\title{
RELACIONES HUMANAS ENTRE EL PROFESIONAL DE ENFERMERÍA Y PACIENTE EN LA SATISFACCIÓN DE LAS NECESIDADES EN EL SERVICIO DE CIRUGÍA DEL HOSPITAL SANTA MARÍA DEL SOCORRO ICA SETIEMBRE 2008 - AGOSTO 2009.
}

\section{Human relations between nursing professional and patient in satisfaction of the needs in the surgery service of Santa María Socorro Hospital of Ica Setiembre 2008 - August 2009.}

\author{
Dra. Isabel Natividad Urure Velazco ${ }^{1 a, d}$; Lic. Enf. Rosario Campos Soto2,a,b; Lic. Enf. Cecilia Teresa Ventura Miranda 3 ,a,c; \\ Est. Tatiana Navarro Quintanilla ${ }^{1, e}$. \\ 1. Facultad de Enfermería. Universidad Nacional San Luis Gonzaga de Ica, Perú \\ 2. Hospital Santa María del Socorro de Ica, Perú \\ 3. Hospital Augusto Hernández Mendoza de Ica, Perú \\ a) Licenciado de Enfermería, b) Magister en Salud Pública, c) Magíster en Ciencias de la Enfermería, d) Doctor en Ciencias de la Salud, \\ e) Estudiante de Enfermería.
}

\section{RESUMEN}

Objetivo: Determinar las relaciones humanas entre el profesional de enfermería y paciente en la satisfacción de necesidades en el servicio de cirugía del Hospital "Santa María del Socorro" de Ica. Material y métodos: Diseño descriptivo - transversal, muestra conformada de 134 pacientes hospitalizados en el servicio de cirugía del Hospital "Santa María del Socorro" de Ica, mayores de 15 años. Como instrumento se utilizó un cuestionario previamente validado con preguntas cerradas de opción múltiple. Para confiabilidad del instrumento se aplicó una prueba piloto al $15 \%$ de la muestra, no incluyéndose los 134 pacientes, permitiendo determinar características finales del instrumento. Se hizo uso del programa de análisis de datos tabulados (EPIDAD 2,1). Resultados: Grupo etáreo predominante fue de 26 a 30 años (17,9\%), siendo el sexo masculino mayoritario con 51,5\%, grado de instrucción secundaria completa y estado civil solteros $32,1 \% ; 38,1 \%$ respectivamente. Las relaciones humanas en la satisfacción de necesidades referente a los principios de la comunicación terapéutica: Interés y permiso fue de muy bueno con un promedio de 2,7 cercano al puntaje esperado que fue 3 , en cuanto a las relaciones humanas de honestidad, coherencia, protección, satisfacción de necesidades sociales y asistencia, resultó bueno con 2,6 los 4 primeros y el último con 2,5 de promedio ponderado; en relación al principio de aceptación, necesidades biológicas y emocionales con puntajes ponderados más bajos 2.3, 2,2 y 2,1 respectivamente poco, adecuado o regular. Conclusiones: Las relaciones humanas entre el profesional de enfermería y paciente en la satisfacción de necesidades resultaron buenas con un promedio general de 2,5.

Palabras clave: Relaciones humanas, profesional de enfermería, paciente.

\section{SUMMARY}

Objective: To determine the human relations between the nursing professional and the patient in the satisfaction of needs in the surgical service of the Hospital "Santa Maria del Socorro" of Ica. Material and methods: Descriptive - transversal design, comprising 134 patients hospitalized in the surgery service of the "Santa Maria del Socorro" Hospital in Ica, aged 15 years and over. As instrument, a questionnaire previously validated with multiple choice closed questions was used. For instrument reliability, a pilot test was applied to $15 \%$ of the sample, not including the 134 patients, allowing to determine final characteristics of the instrument. The tabulated data analysis program (EPIDAD 2.1) was used. Results: The predominant age group was 26 to 30 years old (17.9\%), with the majority male sex with $51.5 \%$, complete secondary education and single marital status $32.1 \% ; 38.1 \%$ respectively. Human relations in the satisfaction of needs concerning the principles of therapeutic communication: Interest and permission was very good with an average of 2.7 close to the expected score that was 3 , in terms of human relations of honesty, consistency, Protection, satisfaction of social needs and assistance, was good with 2.6 the first 4 and the last with 2.5 weighted average; In relation to the principle of acceptance, biological and emotional needs with lower weighted scores 2.3, 2.2 and 2.1 respectively, little, adequate or regular. Conclusions: The human relations between the nursing professional and the patient in the satisfaction of needs were good with a general average of 2.5 .

Key words: Human relations, nursing professional, patient. 


\section{INTRODUCCIÓN:}

La importancia de las relaciones interpersonales del personal de un servicio de salud es un factor que contribuye a determinar la calidad de atención, considerando que las relaciones humanas es de vital importancia para la presencia de un clima organizacional adecuado que permite el desempeño laboral. En efecto las relaciones interpersonales constituyen un papel crítico en el centro hospitalario aunque la calidad de las relaciones interpersonales en sí no basta para incrementar la productividad pero si puede contribuir significativamente.

Enfermería es un campo laboral donde la ayuda y conocimientos permitirán proporcionar bienestar y cuidados al ser humano, con un objetivo primordial que es la satisfacción del paciente; la participación de enfermería es la expresión de ayuda donde el paciente deber ser considerado como un ser individual con problemas y necesidades. Por tal motivo los profesionales de Enfermera deben mantener relaciones humanas basadas en el bienestar, comunicación y aceptación hacia los pacientes; así mismo el respeto por la vida, dignidad y los derechos del ser humano sin importar raza, color, edad, sexo y condición social.

La enfermera es un ser capaz de entender su propia conducta para luego entender a otras personas y ayudarlas a identificar sus necesidades y dificultades, aplicando los principios de relaciones humanas a los problemas que surgen en cualquier nivel de experiencia y de ésta manera cubrir las expectativas que el paciente tiene.

El ser humano es social por naturaleza, y la comunicación constituye la base de todas las relaciones interpersonales, la capacidad para comunicarse eficazmente constituye una habilidad indispensable, y tanto más en aquellos trabajos, como el de enfermería en los que el contacto con los demás es la esencia misma de la labor profesional. (1)

El elemento principal de la interacción entre los seres humanos es la comunicación que permite a los individuos establecer, mantener y mejorar sus relaciones con los demás y con el mundo que les rodea, siendo la comunicación parte importante de la práctica de enfermería basada en una "actitud comunicativa" por lo que el profesional de enfermería debe estar adiestrado en el uso de técnicas de comunicación dirigida al desarrollo de relaciones terapéuticas de trabajo, con el objeto de establecer una comunicación eficaz con el paciente. El fracaso en la comunicación puede originar problemas serios y puede amenazar la credibilidad profesional (2).

El abordaje de esta temática es de importancia como lo demuestran estudios donde se observa brechas existentes y deficiencias en la habilidad de la comunicación enfermerapaciente, el cual influye en la calidad de atención de enfermería. En este sentido se señala a nivel internacional en Valencia, España 2007, el estudio ejecutado por Muñoz $\mathrm{P}$; Soriano E; llegaron a una de las conclusiones que las relaciones interpersonales del personal de enfermería son en general regular, además que las relaciones interpersonales influyen en la calidad de atención al paciente... (3)

En Lima, Perú en 1997, en un estudio de investigación, refieren que el $81 \%$ de la atención es regular cifra significativa que perjudican al establecer relaciones humanas con el usuario... (4).

Considerando" que el personaje central de los servicios asistenciales es por supuesto el paciente. Cuando éste se presenta en el hospital o cualquier otra institución asistencial con una o más problemas de salud (es cada vez mayor el número de pacientes que sufren varios trastornos a la vez); también lo hace como persona, miembro de una familia $y$ ciudadano de un país. Según sea el problema, las necesidades de los pacientes varían con las circunstancias relacionadas y las experiencias anteriores. Una de las funciones importantes es identificar las necesidades inmediatas del paciente y tomar las medidas que satisfagan estas necesidades. Es adecuado utilizar un enfoque de necesidades básicas para los cuidados de enfermería" (5). Justificándose el estudio porque nos permitió conocer y proporcionar a la institución datos 
que nos van a encaminar a mejorar y generar cambios que contribuyen hacia el mantenimiento de las relaciones humanas entre enfermera y paciente, favoreciendo la calidad de atención de enfermería. Señalándose como objetivo determinar las relaciones humanas entre el profesional de enfermería y paciente en la satisfacción de necesidades en el servicio de cirugía del Hospital Santa María del Socorro de Ica.

\section{MATERIAL Y MÉTODOS.}

El estudio utilizó el método descriptivo prospectivo de corte transversal. Siendo el área de estudio ubicado en la Calle Castrovirreyna $\mathrm{N}^{\circ} 759$ en el departamento y provincia de Ica, país Perú. La muestra estuvo conformada por 134 pacientes hospitalizados en el servicio de cirugía, obteniéndose esta muestra aplicándose una fórmula para población finita, mayores de 15 años conscientes, tomando como criterios de inclusión y excluyéndose a pacientes analfabetos e inconscientes. Se utilizó un cuestionario que fue estructurado de 2 partes. Primera parte: Referente a Datos Generales (edad, sexo, grado de instrucción, estado civil) y la Segunda parte: correspondiente a reactivos relativos a las relaciones humanas entre el profesional de enfermería y paciente en la satisfacción de las necesidades distribuidos en las 7 dimensiones de la comunicación terapéutica (aceptación, interés, honestidad, coherencia, asistencia, permiso y protección), con 17 reactivos; y 3 dimensiones de las necesidades: Biológicas, emocionales (seguridad y protección) y sociales (amor y pertenencia) con 11 reactivos. Utilizándose 28 reactivos con una escala de alternativas cuyas puntuaciones fueron 1 (nunca), 2 (a veces), 3 (siempre).

En la recolección de datos se utilizó como técnica la encuesta y como instrumento un cuestionario que fue elaborado teniendo como base el instrumento utilizado por Mogollón (6) en Maracay, Venezuela, con preguntas cerradas de opción múltiple el cual fue validado y sometido a juicio de expertos, realizándose una prueba piloto al $15 \%$ de la muestra en estudio para evaluar la confiabilidad del instrumento, no incluyéndose los 134 pacientes, permitiendo determinar las características finales del instrumento. Se hizo uso del programa de análisis de datos tabulados EPIDAT 2,1.

\section{RESULTADOS:}

Al finalizar el procesamiento de datos se elaboraron tablas que muestran el grupo etáreo predominante fue de 26 - 30 años el $17,9 \%$ y $16-20$ años el $14,9 \%$. Tabla 1 , siendo el sexo masculino el mayoritario con $51,5 \%$ Tabla 2 , predominando el grado de instrucción secundaria completa con 32,1\% (43 pacientes) Tabla 3, la mayoría solteros con 38,1\% (51 pacientes) Tabla 4. En cuanto a los principios de la comunicación los promedios ponderados que se han acercado a la valoración optima de 3 es interés y permiso con 2,7 considerando como muy bueno, lo que indica interés por atenderlo y cuidado de solicitar permiso al efectuar un procedimiento. La honestidad, coherencia, protección y necesidades sociales con 2,6 de promedio ponderado calificado como bueno lo que muestra una comunicación asertiva, usando un vocabulario que facilite el dialogo con el paciente, y se observa que protegen al enfermo al colocar inyección y/o suero limpiando la piel con algodón y alcohol con antelación, preocupándose como se siente, relacionándose con su familia y orienta acerca de sus necesidades. El rubro asistencia con 2,5 podría también considerarse como bueno lo que indica que la enfermera se comunica con el paciente para conocer sus necesidades y verificar los cuidados brindados. En cuanto a las necesidades emocionales, biológicas y aceptación con puntajes más bajos 2,1,2,2 y 2.3 respectivamente recae en la alternativa "a veces" inferior al promedio general que es de 2,5 , mostrando que a veces la enfermera muestra gestos o es amable ante incomodidad del enfermo por dolor, o cuando controla los signos vitales, cambios de posición, sueño y descanso, al realizar la valoración física, al controlar la venoclisis y/o drenaje, o conocer sus creencias religiosas dialoga a veces con el enfermo. Tabla 
Tabla $\mathrm{N}^{\circ}$ 1. Relaciones humanas entre el profesional de enfermería y paciente en la satisfacción de necesidades del servicio de cirugía. Hospital "Santa María del Socorro" de Ica. Por grupo de edad

\begin{tabular}{|c|c|c|}
\hline $\begin{array}{l}\text { Grupo de } \\
\text { edad }\end{array}$ & $\begin{array}{c}\mathbf{N}^{\circ} \mathrm{de} \\
\text { Pacientes }\end{array}$ & $\%$ \\
\hline $16-20$ & 20 & 14,9 \\
\hline $21-25$ & 19 & 14,2 \\
\hline $26-30$ & 24 & 17,9 \\
\hline $31-35$ & 14 & 10,4 \\
\hline $36-40$ & 17 & 12,7 \\
\hline $41-45$ & 9 & 6,7 \\
\hline $46-50$ & 9 & 6,7 \\
\hline $51-55$ & 5 & 3,7 \\
\hline $56-60$ & 9 & 6,7 \\
\hline $61-65$ & 4 & 3,0 \\
\hline 66 y más & 4 & 3,0 \\
\hline Total & 134 & 100.0 \\
\hline
\end{tabular}

Tabla $\mathbf{N}^{\circ} 2$. . Relaciones humanas entre el profesional de enfermería y paciente en la satisfacción de necesidades del servicio de cirugía. Hospital "Santa María del Socorro" de Ica. Por Sexo

\begin{tabular}{lcc}
\hline \multicolumn{1}{c}{ Sexo } & \multicolumn{2}{c}{$\begin{array}{c}\mathbf{N}^{\circ} \text { de } \\
\text { Pacientes }\end{array}$} \\
\hline Masculino & 69 & 51,5 \\
\hline Femenino & 65 & 48,5 \\
\hline Total & 134 & 100,0 \\
\hline \multicolumn{4}{c}{ Fuente: } & Base de datos
\end{tabular}

Tabla $N^{\circ}$ 3. . Relaciones humanas entre el profesional de enfermería y paciente en la satisfacción de necesidades del servicio de cirugía. Hospital "Santa María del Socorro" de Ica. Por Grado de Instrucción

\begin{tabular}{|c|c|c|}
\hline $\begin{array}{l}\text { Grado de } \\
\text { instrucción }\end{array}$ & $\begin{array}{c}\mathbf{N}^{\circ} \text { de } \\
\text { Pacientes }\end{array}$ & $\%$ \\
\hline Primarialncompleta & 8 & 6 \\
\hline PrimariaCompleta & 6 & 4,5 \\
\hline Secundarialncompleta & 25 & 18,7 \\
\hline SecundariaCompleta & 43 & 32,1 \\
\hline SuperiorIncompleta & 21 & 15,7 \\
\hline SuperiorCompleta & 30 & 22,4 \\
\hline Ninguno & 1 & 9,7 \\
\hline Total & 134 & 100,0 \\
\hline
\end{tabular}

Tabla $N^{\circ} 4$. . Relaciones humanas entre el profesional de enfermería y paciente en la satisfacción de necesidades del servicio de cirugía. Hospital "Santa María del Socorro" de Ica. Por estado civil

\begin{tabular}{lcc}
\hline $\begin{array}{c}\text { Estado } \\
\text { Civil }\end{array}$ & $\begin{array}{c}\mathbf{N}^{\circ} \text { de } \\
\text { Pacientes }\end{array}$ & $\%$ \\
\hline Soltero & 51 & 38,1 \\
\hline Casado & 36 & 26,9 \\
\hline Viudo & 42 & 31,3 \\
\hline Divorciado & 3 & 2,2 \\
\hline Viudo & 2 & 1,5 \\
\hline Total & 134 & 100,0 \\
\hline
\end{tabular}

Fuente: Base de datos
TABLA 5. Promedio ponderado según rubro de las Relaciones Humanas entre el profesional de enfermería y paciente en la Satisfacción de las Necesidades, Servicio de Cirugía, Hospital Santa María del Socorro de Ica.

\begin{tabular}{llc}
\hline \multicolumn{1}{c}{ Rubro } & $\begin{array}{c}\text { Promedio } \\
\text { ponderado }\end{array}$ \\
\hline a) & Aceptación & 2,3 \\
\hline b) Interés & 2,7 \\
\hline c) & Honestidad & 2,6 \\
\hline d) & Coherencia & 2,6 \\
\hline e) asistencia & 2,5 \\
\hline f) & permiso & 2,7 \\
\hline g) Protección & 2,6 \\
\hline h) Necesidadesbiológicas & 2,2 \\
\hline i) & Necesidadesemocionales & 2,1 \\
\hline j) & Necesidades sociales & 2,6 \\
\hline Promedio ponderado general & 2,5 \\
\hline \multicolumn{2}{c}{ Fuente: base de datos } \\
\end{tabular}

\section{DISCUSIÓN:}

Las relaciones es un conjunto de mecanismos de comunicación de un grupo de personas, en el caso del profesional de enfermería constituye una herramienta mediante la cual identifica las necesidades del enfermo y ayuda en la satisfacción.

El estudio alcanzó un promedio ponderado general de 2,5 con una brecha existente de 0,5 por alcanzar el promedio óptimo de 3 por consolidar y fortalecer las relaciones humanas del profesional de enfermería con el paciente orientado a la satisfacción de las necesidades básicas utilizando la comunicación asertiva.

El nivel educativo de los pacientes hospitalizados objeto de estudio fue relativamente alto $32,1 \%$ secundaria completa, $22,4 \%$ instrucción superior, lo que determina que a mayor grado de instrucción es mayor el compromiso profesional en la interacción enfermera/paciente, asegurando la confiabilidad del instrumento. Las relaciones humanas del profesional de enfermería con el paciente en la satisfacción de las necesidades, respecto al indicador de la comunicación terapéutica aceptación fue regular con un promedio ponderado 2,3 evidenciando la poca importancia del diálogo en el aspecto espiritual, enfocando más los cuidados dirigidos a los procedimientos técnicos en desmedro de la interacción enfermerapaciente. Este hallazgo es coincidente con Mogollón C. en Venezuela, cuyo resultado fue en promedio $50 \%$ cuya diferencia $50 \%$ 
reconoce las diferencias personales, valora al enfermo, respeta las creencias personales y toma en cuentas las opiniones del enfermo (6). Referente a los indicadores interés y permiso, el estudio mostró una relación interpersonal muy buena de la enfermera con el paciente, alcanzando en ambos rubros 2,7 cercano al puntaje esperado que es de 3 con una brecha mínima de 0,3 lo que denota la preocupación del profesional de enfermería por velar por el bienestar del paciente, mostrando interés por escucharlo, solicitando permiso para efectuar cualquier procedimiento, coincidente con Mogollón en el principio terapéutico permiso quien señala que "lo aplican de forma satisfactoria el $65 \%$ de los profesionales (6), solicitando el consentimiento e informe a los enfermos de los procedimientos a realizar.

Sin embargo no mostrando coincidencia con el estudio en el principio de interés donde el autor antes mencionado señala que "solo el $50 \%$ ) muestra preocupación por establecer la comunicación (6). En relación a los principios de la comunicación terapéutica honestidad, coherencia, protección y asistencia, aquí mostraron las relaciones humanas de la enfermera con el paciente en la satisfacción de las necesidades bueno, alcanzando un promedio de 2,6 los 3 primeros y el último (asistencia) 2,5 con una brecha de 0,4 lo que demuestra que hay buena comunicación entre enfermera-paciente entablando relación receptiva y veraz con el enfermo, usando expresiones verbales coherentes, verifican la satisfacción de los cuidados brindados, así como uso de medidas de protección. Mostrando inconsistencia los resultados con Mogollón (6) en relación a los principios de honestidad, coherencia y asistencia, encontrando limitaciones y deficiencias en la receptividad ante las necesidades de los mismos al comunicarse con los enfermos, al expresarse de manera precisa con un vocabulario adecuado, dedicarle tiempo, etc, alcanzando un promedio de $40 \%$ los 2 primeros y el último $50 \%$ (asistencia). Pero mostrando consistencia con el principio de protección un promedio de $55 \%$ (6). Respecto a las necesidades biológicas aquí mostró que las relaciones humanas de la enfermera con el enfermo son poco adecuados o regulares en la satisfacción de las necesidades básicas alcanzando un promedio de 2,2 con un déficit existente de 0,8 , enfocado más la asistencia en procedimientos técnicos restando importancia a la comunicación terapéutica (enfermera/paciente), herramienta fundamental en la satisfacción de necesidades básicas. Se observó los resultados similares con el estudio de Mogollón en Venezuela en la que concluye que "se distinguió el mayor porcentaje de No lo hizo, $53 \%$ (6) referente a las necesidades emocionales en las 3 premisas alcanzó el promedio ponderado de 2,1 mostrando que las relaciones humanas entre enfermera y paciente fueron regulares, denotando que a veces muestra gestos, palabras de afecto para darle apoyo o se muestra amable cuando se sintió incómodo o tenía dolor. Siendo coincidente este hallazgo con el estudio de Oré $\mathrm{H}$, donde concluye que "el $81 \%$ refieren que la atención es regular, cifra significativa que perjudican al establecer las relaciones humanas con el usuario" (4). Asimismo es consistente con Mogollón en relación a las necesidades emocionales, alcanzando el promedio $63 \%$ se ubicó en No, por tanto no son satisfechos por la totalidad de los profesionales de enfermería (6).

En la satisfacción de las necesidades sociales, aquí mostró el estudio efectuado buenas relaciones humanas, alcanzando el promedio en las 4 premisas 2,6, que influye en elevar la autoestima del paciente, sintiéndose útil e importante, favoreciendo la comunicación terapéutica del enfermo. Este hallazgo coincide con el estudio de Mogollón en la que concluye que "se apreció que un $60 \%$ proporcionaban los cuidados especiales e informaban a los familiares del enfermo" (6). Se puede señalar que la muestra estudiada en las 10 dimensiones: referente a los 7 principios de la comunicación terapéutica (aceptación, interés, honestidad, coherencia, asistencia, permiso, protección) y las 3 necesidades (biológicas, emocionales y sociales) alcanzó como promedio ponderado general de 2,5 de un rango o puntaje de 1 al 3 , correspondiendo a buenas relaciones humanas entre enfermera 
y paciente. Este resultado no es consistente con el estudio de Muñoz en la que concluye que las relaciones interpersonales del personal de enfermería son en general regulares, además que las relaciones interpersonales influyen en la calidad de atención al paciente crónico del Hospital Militar Central (3).

\section{CONCLUSIONES.}

Las relaciones humanas entre el profesional de enfermería y paciente en la satisfacción de necesidades en el servicio de cirugía del Hospital Santa María del Socorro fueron buenas con un promedio general de 2,5. Existiendo todavía una brecha de 0,5 por alcanzar el rango óptimo de 3, y favorecer buenas relaciones humanas enfermerapaciente.

\section{RECOMENDACIONES.}

Propiciar y fomentar a los profesionales de enfermería a través de talleres, trabajos grupales, continuos las buenas relaciones interpersonales con el enfermo, estrategias que mantengan motivadas la interacción eficaz y superar la brecha mínima existente, conducente a brindar calidad de atención de enfermería.

\section{Correspondencia}

Dra. Isabel Natividad Urure Velazco

Correo electrónico: isabelnauv@yahoo.com

\section{REFERENCIAS BIBLIOGRÁFICAS.}

1. Diez MI, Fernández JL, López $\mathrm{S}$, Martin R, Martínez A, Romero M. et al. Manual de Enfermería. Ed Lexus. Barcelona, España: 2003

2. Potter $\mathbf{P}$. Fundamentos de Enfermería Teoría y Práctica. $3^{\underline{a}}$ ed. Madrid, España: Ed. Harcourt Brace: 2000.

3. Muñoz $\mathbf{P}$, Soriano E. Relaciones Interpersonales del Personal de Enfermería y su Influencia en la Calidad de Atención del Paciente Coronario Tesis Hospital Militar Central: Valencia, España 2007.
4. Ore H. Relaciones Humanas entre el Profesional de Enfermería y Usuario en los Servicios de Medicina y Cirugía en el Hospital Hipólito Unanue. Tesis de Pregrado. Lima, Perú. Facultad de Enfermería, Universidad Privada San Martín de Porres; 1997.

5. Brunner y Suddarth. Enfermería Médico Quirúrgico. $7^{\mathrm{a}}$ ed. México. Ed. Interamericana: 1998 p. 15.

6. Mogollón C., Pinto Z. Comunicación Terapéutica en la Satisfacción de las Necesidades de los Enfermos en el Postoperatorio Inmediato (Internet) Maracay, Venezuela (Citado el 15 de Enero 2008) Disponible en: http://www.monografias.com/trabajos.s/po stoperatoria.shtml.

Recibido: 18/12/2015

Aprobado para publicación: 15/04/16 


\section{INSTRUCCIONES PARA LA PRESENTACIÓN DE ARTÍCULOS DE LA REVISTA: "ENFERMERÍA A LA VANGUARDIA"}

La revista científica "Enfermería a la Vanguardia" es una publicación editada por la Facultad de Enfermería de la Universidad Nacional "San Luis Gonzaga" de Ica (UNICA). Tendrá una periodicidad semestral y se publicará en su versión impresa como digital.

La revista REVAN recepcionará y publicará artículos completos originales, inéditos en temas relacionados en el campo de enfermería, en el contexto Biopsicosocial del ser humano y otras disciplinas, con el fin de contribuir a mejorar la situación de salud del país y de la región. El envío de los artículos debe ser presentado, grabado en CD a la Facultad de Enfermería (mesa de partes) o por correo electrónico a:

revistaenfermerialavanguardia@unica.edu.pe acompañando:

\section{CARTA DE PRESENTACIÓN.}

Dirigida a la Directora de la Revista, firmada por el autor responsable, solicitando la evaluación del artículo para que sea revisado y editado para su publicación, determinando el tipo de artículo y autor(es).

\section{UECLARACIUN JURAUA UE SER AU IUR} Y AUTORIZACIÓN DE PUBLICACIÓN.

Firmada por todos los autores, declarando que el contenido del artículo presentado es de propiedad y responsabilidad exclusiva de los autores, no habiendo sido publicado en forma parcial o completa en otras revistas, otorgando los derechos de autor a la revista "Enfermería a la Vanguardia" para su publicación.

\section{NORMAS GENERALES.}

Todo artículo presentado a la REVAN debe ser escrito en español, tratar temas de interés en

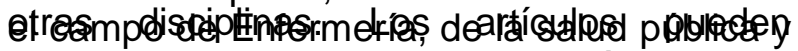
pertenecer a las siguientes categorías:

- Editorial

- Artículos originales

- Artículos breves

- Artículos de revisión.

$$
\text { Cartas, al editor }
$$

PREPARACION DE LOS ARTÍCULOS.

- Todo el texto debe ser redactado en el programa Word para Windows XP o Vista a doble espacio en letra Arial 12, formato A4 con márgenes de $3 \mathrm{~cm}$.

- La Primera página debe incluir: Título del artículo con una extensión de hasta 15 palabras, en español e inglés.

Nombres de los autores como desean que aparezcan en el artículo, su filiación institucional, ciudad, país, profesión y grado académico.

- Nombre del autor corresponsal, indicando su dirección, teléfono y correo electrónico.

- Incluir declaración en caso exista fuente de financiamiento y conflictos de interés.

- En caso el estudio haya sido presentado como resumen a un congreso o es parte de una tesis debe precisarlo con la cita correspondiente. Agradecimientos cuando corresponda, debe mencionarse en forma específica a quién y por qué tipo de apoyo en la investigación se realiza el agradecimiento.

Para el resto de páginas debe considerarse los siguientes aspectos.

- Cada sección del artículo empieza en una nueva página.

- La presentación de resultados debe ir acompañada de tablas o figuras insertadas al final del articulo ordenados con números arábigos y remitidos adjuntos en Microsoft Excel con sus respectivas leyendas; Las tablas deben tener sólo líneas horizontales para separar el encabezado del cuerpo de la tabla; Las figuras (gráficos estadísticos), imágenes o mapas deben ser grabados en formato JPG a una resolución mayor de 600 dpi o 300 pixeles.

- El número de tablas, gráficos y figuras

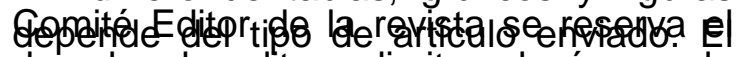
derecho de editar y limitar el número de éstas.

- Las fracciones se deben separar de los números enteros con coma decimal, seguido de dos decimales.

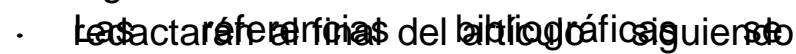
las normas Vancouver (www. icmje.org/index.html), serán únicamente las que han sido citadas en el 
artículo, se ordenarán correlativamente según su aparición. Estas llamadas de cita precedidas de un espacio, se colocan entre paréntesis antes del punto, coma u otro signo de puntuación, ejemplo: (1) o $(2,5)$.

Los autores deberán ser resaltados en "negrita"; en el caso de existir más de seis autores, deberán colocarse los seis primeros autores seguidos de et. al., separados por una coma. Ejemplos.

Palomino M, Villaseca P, Cárdenas F, Ancca J, Pinto M. Eficacia y residualidad de dos insecticidas piretroides contra Triatoma infestans en tres tipos de vivienda. Evaluación de campo en Arequipa, Perú. Rev Perú Med Exp Salud Pública. 2008; 25(1): 9-16.

Troyes L, Fuentes L, Troyes M, Canelo L, García M, Anaya E, et al. Etiología del síndrome febril agudo en la provincia de Jaén, Perú 2004-2005. Rev Perú Med Exp Salud Pública. 2006; 23(1): 5-11.

\section{Libro:}

Acha P, Szyfres B. Zoonosis y enfermedades transmisibles comunes al hombre y los animales. $3 a$ ed. Washington DC: Organización Panamericana de la Salud; 2003.

\section{Capítulo de libro:}

Farmer J. Enterobacteriaceae: Introduction and identification. En: Murray PR, Barón EJ, Pfaller MA, Tenover FC, Yolken RH, editors. Manual of clinical microbiology. 7th ed. Washington DC: American Society for Microbiology; 1999. p. 442 -58.

\section{Tesis:}

Pesce H. La epidemiología de la lepra en el Perú. Tesis Doctoral. Lima, Perú. Facultad de Medicina, Universidad Nacional Mayor de San Marcos; 1961.45 pp.

\section{Página web:}

Brasil, Ministerio da Saúde. Sistema de Informacoes sobre Ornamentos Públicos em Saúde (Internet). Brasilia: Ministerio da Saúde; 2009. (Citado el 12 de Mayo 2009) Disponible en: http://portal.saude.gov.br/ portal/se/area.cfm7id area $=572$.

\section{NORMAS ESPECÍFICAS POR TIPO DE ARTÍCULO.}

\section{EDITORIALES.}

Son a solicitud del Comité Editor de la REVAN.

\section{ARTÍCULOS ORIGINALES.}

Sólo son productos de investigación los cuales deberán estar redactados hasta en 15 páginas (incluye tablas y gráficos) según el siguiente esquema.

Resumen: en español e inglés. No debe contener más de 250 palabras. Este resumen debe incluir de manera concisa: objetivos, materiales y métodos, resultados y conclusiones.

- Palabras clave: de tres hasta siete, las cuales deben basarse en descriptores en ciencias de la salud, para español el DeCS de BIREME (Disponible en: http.//decs.bvs.br/E/ decswebe.htm) y en inglés el MeSH de la NLM (Disponible en: www.pubmed.gov).

Introducción: Exposición breve (máximo dos páginas) de la situación actual del problema, antecedentes, justificación y objetivo del estudio.

Materiales y métodos: Se describe la metodología usada de tal forma que permita la reproducción del estudio y la evaluación de la calidad de la información por los lectores y revisores. Se debe describir el tipo de estudio, las características de la población y forma de selección de la muestra cuando sea necesario. En algunos casos, es conveniente describir el área de estudio. Cuando se usen plantas medicinales, describir los procedimientos de recolección e identificación. Precisar la forma cómo se midieron o definieron las variables de interés.

Detallar los procedimientos realizados, si han sido previamente descritos, hacer la cita correspondiente. Mencionar los procedimientos estadísticos empleados.

Detallar los aspectos éticos involucrados en la realización del estudio, como la aprobación por un Comité de Ética Institucional, el uso de consentimiento Informado, entre otras.

- Resultados: la presentación de los hallazgos, debe ser en forma clara, sin opiniones ni interpretaciones, salvo, en las de alcance estadístico. Se pueden complementar hasta con ocho tablas 0 figuras, las cuales no deben repetir la información que está en texto.

- Discusión: se debe interpretar los resultados, comparándolos con los hallazgos de otros autores, exponiendo las 
sugerencias, postulados o conclusiones a las que llegue el autor. Debe incluirse las limitaciones que hubiera en el estudio.

- Agradecimientos: cuando corresponda, debe mencionarse en forma específica a quién y por qué tipo de apoyo en la investigación se realiza el agradecimiento.

- Conflictos de interés: debe mencionarse si existe algún conflicto de interés.

- Referencias bibliográficas: siguiendo el estilo Vancouver en número no mayor de 30 referencias.

\section{ORIGINALES BREVES.}

Estos artículos son el resultado de investigaciones, pueden incluirse también reporte de brotes o avances preliminares, de investigación que por su impacto requieren una rápida publicación, estos deberán ser redactados hasta en 2000 palabras (sin contar resumen, referencias, tablas y figuras). El resumen en español e inglés es no estructurado, tiene un límite de 150 palabras con 3 a 5 palabras claves: Contiene una introducción, material y métodos, resultados, pudiéndose incluir hasta 4 tablas o figuras, discusión, y no más de 15 referencias bibliográficas.

\section{ARTíCULOS DE REVISIÓN.}

Trabajos que constituyen una exhaustiva revisión del tema de investigación del autor, se incluyen tesis, revisiones taxonómicas y recapitulaciones, sobre temas actuales de interés en el campo de enfermería y otras disciplinas. La estructura del artículo queda a criterio del autor, el $\mathrm{N}^{\circ}$ de páginas no debe exceder de 25 páginas sin contar tablas 0 figuras que no serán más de 10. Debe incluir un resumen en español - inglés, no estructurado con un límite de 150 palabras con 3 - 5 palabras clave. Podrán utilizar hasta 100 referencias bibliográficas.

\section{CARTA AL EDITOR.}

Esta sección está abierta para todos los lectores de la REVAN a la que puede enviar sus preguntas, comentarios y críticas a los artículos que hayan sido publicados en los últimos números, teniendo en cuenta la posibilidad de que los autores aludidos puedan responder. Podrá aceptarse la comunicación de investigaciones preliminares 0 intervenciones de brotes que no hayan sido publicados ni sometidos a publicaciones en otras revistas, así como algunos comentarios sobre problemas de salud pública, ética y otras disciplinas. La extensión máxima aceptable es de 1.000 palabras sin contar referencias, tablas y figuras, con un máximo de 6 referencias bibliográficas (incluyendo la referencia del artículo que la motivó, cuando sea el caso) y una tabla y figura. 


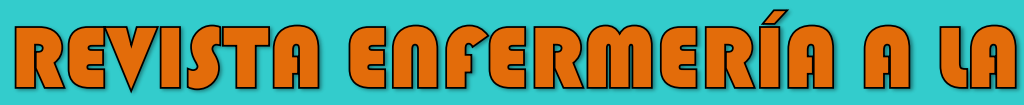

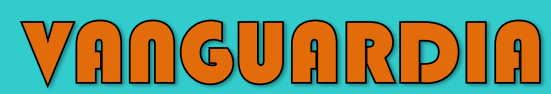

VOLUMEN 4 NÚMERO 1. ENERO - JUNIO 2016 / VOLUME 4 NUMBER 1. JANUARY - JUNE 2016

\section{EDITORIAL/EDITORIAL}

\section{LA ÉTICA Y VALORES EN ENFERMERÍA}

Ethics and Values in Nursing

Dra. Isabel Natividad Urure Velazco.......

\section{ARTÍCULO ORIGINAL/ORIGINAL ARTICLE}

RELACIÓN ENTRE LAS CARACTERÍSTICAS SOCIODEMOGRÁFICAS LABORALES CON LAS DIMENSIONES DE LA FELICIDAD EN LOS PROFESIONALES DE LA PROVINCIA DE TARMA-2015

Relationship between working sociodemographic characteristics with the dimensions of happiness in professionals from Tarma Province - 2015

Santos-López, Elisabet Sara, Hinostroza Robles, Nelly Marleni, Orihuela Espinoza, Rosa Clara, Enriquez Gonzáles, Giovanna Betsabé, Gora Atencio, Marlene Deysi, Córdova-Baldeón, Isaac Sicilio.

TRASCENDENCIA DE LA ENSEÑANZA DEL CUIDADO ENFERMERO Y SU REPERCUCION SOCIAL REGION SUR PERÚ 2012 - 2013

Trascendence Of The Nursing Care Teaching And Its Social Impact Region Sur Perú 2012 2013

Sonia Velásquez Rondón

CUIDADO DE ENFERMERÍA Y SATISFACCIÓN DEL ADULTO MAYOR, HOSPITAL SANTA MARIA DEL SOCORRO DE ICA-2014

Nursing care and satisfaction of elderly Hospital Santa Maria Socorro Ica-2014

Susana Alvarado Alfaro

RELACIONES HUMANAS ENTRE EL PROFESIONAL DE ENFERMERIAA Y PACIENTE EN LA SATISFACCIÓN DE LAS NECESIDADES EN EL SERVICIO DE CIRUGÍA DEL HOSPITAL SANTA MARÍA DEL SOCORRO ICA SETIEMBRE 2008 - AGOSTO 2009.

Human relations between nursing professional and patient in satisfaction of the needs in the surgery service of Santa María Socorro Hospital of Ica Setiembre 2008 - August 2009.

Isabel Natividad Urure Velazco; Rosario Campos Soto, Cecilia Teresa Ventura Miranda' Est. Tatiana Navarro Quintanilla..... 


\section{Impreso por Editorial Universitaria Universidad}

Nacional San Luis Gonzaga de Ica (UNICA)

Fecha de Impresión: Junio del 2016 